\title{
Aplicação das equações diferenciais de momento e energia na obtenção da perda de potência em mancais de deslizamento
}

\author{
Gabriel, A. C.; Zanete, O. Z.; Gonçalves, J. B.; Monhol F. A. F.* \\ Engenharia Mecânica, Instituto Federal do Espirito Santo, São Mateus, Brazil.
}

* e-mail: filipe.monhol@ifes.edu.br

\begin{abstract}
Resumo
Foi avaliada a perda de potência em um mancal de deslizamento associada a diferentes tipos de lubrificantes. A perda de potência foi estimada através da resolução das equações da continuidade, da quantidade de movimento e conservação de energia aplicadas ao sistema em questão. Os resultados mostraram influência do lubrificante na perda de potência do mancal. Alguns dos óleos apresentaram redução de até $30 \%$ da perda de potência quando comparado ao óleo recomendado pelo fabricante.
\end{abstract}

\begin{abstract}
This study evaluated the power loss in the bearing by comparing different lubricants. The power loss was estimated by solving the heat transfer equations such as continuity, momentum e energy conservation applied to the system in question. The results showed influence of lubricant viscosity on the bearing power loss. Some oils analyzed decreased the power loss by up to $30 \%$ compared to the manufacturer recommended oil.
\end{abstract}

Keywords (Palavras chaves): Equações diferenciais, Perda de potência, Mancais, Dissipação viscosa.

\section{Introdução}

Os mancais de deslizamento são conhecidos também como mancais lubrificados hidrodinamicamente e estão presentes em muitas operações modernas, especialmente em operações que exigem maior precisão. Estes são geralmente representados por superfícies cilíndricas, alojando um eixo em seu interior. Alves [1], define lubrificação como a aplicação de um filme de alguma substância, geralmente um fluido lubrificante, com a função de separar superfícies em contato, diminuindo o atrito entre as partes sólidas, além de reduzir a temperatura dos acoplamentos e inibir a corrosão, entre outras funções. De acordo com [2], a inserção de um lubrificante na folga entre eixo e mancal pode reduzir em até 1000 vezes o atrito entre os elementos quando do contato metal-metal. Em escoamentos de alta velocidade, a dissipação viscosa se apresenta de maneira dominante. Provocando a conversão da energia cinética do fluido em energia térmica [3]. Devido a dissipação viscosa, quanto menor for o valor da viscosidade do lubrificante menor será a perda de potência. Com base nessa análise, foi estudada a perda de potência para diferentes óleos lubrificantes, objetivando encontrar um lubrificante que diminua este valor de potência dissipada.

\section{Metodologia}

\subsection{Modelagem do problema}

Segundo [3], o escoamento de óleo em mancal pode ser aproximado por um escoamento entre duas placas paralelas. As seguintes hipótese foram adotadas: regime permanente, fluido incompressível, propriedades constantes e forças de corpo desprezíveis. Aplicando as equações da continuidade (Eq. 1), da quantidade de movimento (Eq. 2) e da conservação da energia (Eq. 3) para o escoamento entre duas placas planas temos:

$$
\begin{aligned}
& \frac{\partial u}{\partial x}+\frac{\partial v}{\partial y}=0 \\
& \rho\left(u \frac{\partial u}{\partial x}+v \frac{\partial u}{\partial y}\right)=\mu \frac{\partial^{2} u}{\partial y^{2}}-\frac{\partial F}{\partial x} \\
& \rho c_{p}\left(u \frac{\partial T}{\partial x}+v \frac{\partial T}{\partial y}\right)=k\left(\frac{\partial^{2} T}{\partial x^{2}}+\frac{\partial^{2} T}{\partial y^{2}}\right)
\end{aligned}
$$

Resolvendo com as devidas simplificações e aplicando as condições de contorno ideias obtém-se a distribuição de temperatura ao longo do filme (Eq. 4). Assim, a perda de potência relacionada a geração de calor pode ser calculada através da Lei Fourier dada pela Eq. 5. 


$$
\begin{aligned}
& T(y)=T_{0}+\frac{\mu V^{2}}{2 k}\left(\frac{y}{L}-\frac{y^{2}}{L^{2}}\right) \\
& \dot{q}=-\left.k \frac{d T}{d y}\right|_{y=0}
\end{aligned}
$$

\subsection{Materiais e métodos}

Foi analisado o mancal de apoio do eixo de suporte da fresa de uma fresadora Modelo FU.301- Série 244. As dimensões do mancal são apresentadas na Tab.1.

\begin{tabular}{l|l|l}
\multicolumn{3}{l}{ Tabela 1: Medidas do mancal de deslizamento do suporte da fresa. } \\
\hline Medida & Valor & Observações \\
\hline D1 & $55,95[\mathrm{~mm}]$ & Diâmetro do eixo \\
\hline D2 & $56,2[\mathrm{~mm}]$ & Diâmetro do Mancal \\
\hline L & $85[\mathrm{~mm}]$ & Comprimento \\
\hline N & 40 à 2000 [rpm] & Rotações Avaliadas
\end{tabular}

O fabricante da fresadora especifica o lubrificante MOBIL VACTRA N ${ }^{\circ} .2$ (MV2). Para o estudo foram escolhidos três óleos SAE cujas propriedades são estimadas por [4]. Além destes, outros três lubrificantes industriais aplicados a lubrificação de máquinas em geral foram avaliados (LU220, LU320 e LU460). Todas as suas propriedades foram obtidas em [5]. A Tab. 2 mostras as propriedades de interesse de cada óleo na temperatura de trabalho que é de $41,5^{\circ} \mathrm{C}$.

Tabela 2: Propriedades dos óleos lubrificantes $\left(41,5^{\circ} \mathrm{C}\right)$.

\begin{tabular}{c|c|c}
\hline Lubrificante & Viscosidade $(\boldsymbol{\mu})$ & Condutividade (k) \\
\cline { 1 - 2 } MV2 & $0,0600[\mathrm{~Pa} \cdot \mathrm{s}]$ & \\
\cline { 1 - 2 } SAE 5W20 & $0,0419[\mathrm{~Pa} \cdot \mathrm{s}]$ & \\
\cline { 1 - 2 } SAE 5W30 & $0,0578[\mathrm{~Pa} \cdot \mathrm{s}]$ & \multirow{2}{*}{$0,14417[\mathrm{~W}(\mathrm{~m} \cdot \mathrm{K})]$} \\
\hline SAE 5W40 & $0,0498[\mathrm{~Pa} \cdot \mathrm{s}]$ & \\
\cline { 1 - 2 } LU 220 & $0,1844[\mathrm{~Pa} \cdot \mathrm{s}]$ & \\
\cline { 1 - 2 } LU 320 & $0,2688[\mathrm{~Pa} \cdot \mathrm{s}]$ & \\
\hline LU 460 & $0,3820[\mathrm{~Pa} \cdot \mathrm{s}]$ & \\
\hline
\end{tabular}

As velocidades de rotação analisadas foram: 40, 50, $63,80,100,125,160,200,250,315,400,500,630$, $800,1000,1250,1600$ e 2000 RPM.

\section{Resultados e discussão}

Como apresentado na Fig. 1, os lubrificantes SAE apresentam reduções significativas da perda de potência (cerca de até $30 \%$ para o SAE 5 W50), quando comparados ao lubrificante determinado pelo fabricante da máquina e aos lubrificantes industriais LU's. Dessa maneira, a aplicação deste tipo de óleo na lubrificação dos mancais analisados, provoca uma melhoria na eficiência do equipamento e consequentemente uma economia nos gastos com fontes energéticas.

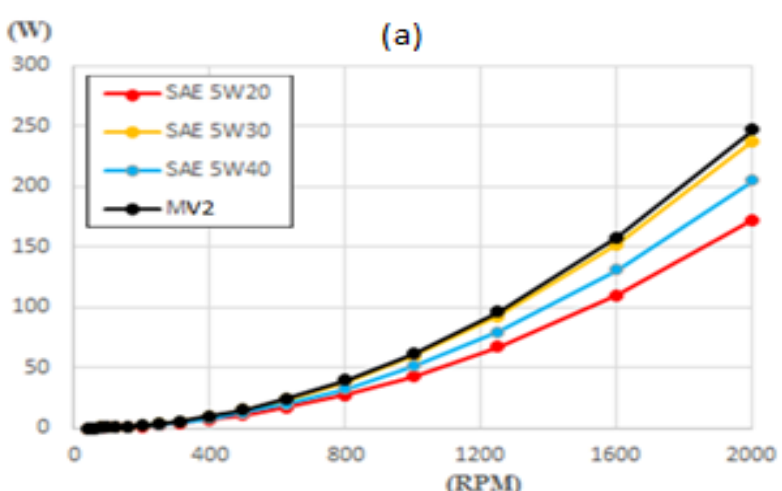

(b)

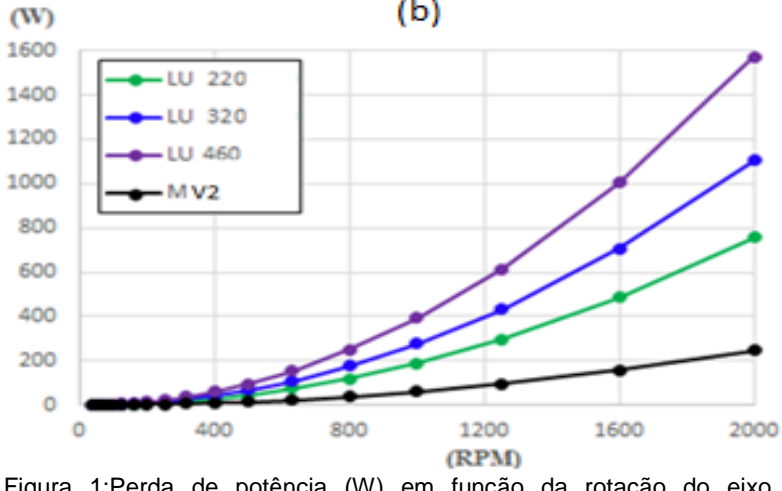

Figura 1:Perda de potência (W) em função da rotação do eixo. (a) Óleos SAE. (b) Óleos LU

\section{Conclusões}

A partir da análise dos resultados, os lubrificantes que apresentaram redução na perda de potência são os óleos SAE 5W, sendo que o lubrificante SAE 5W20 o óleo que apresenta reduções mais significativas (cerca de $30 \%$ ). Portanto deve ser verificada a viabilidade da substituição do óleo lubrificante recomendado pelo fabricante por qualquer um dos óleos SAE 5W.

\section{Referências}

[1] Alves, D.S. "Investigação do Efeito Térmico no Comportamento Dinâmico de Mancais Hidrodinâmicos". Dissertação Mestrado. Universidade Estadual de Campinas, Campinas - SP. 2011.

[2] Antonic, Z., Nikolic, N., Radomirovic, D. Mechanism and Machine Theory, Vol. 46, pp. 975-985, 2011.

[3] Cengel,Y.A., Ghajar, A.J. Transferência de calor e massa. 4. Ed. São Paulo: AMGH Editora, 2011.

[4] Canciam, C.A., Revista Universidade Vale do Rio Verde, Três Corações, Vol.11 (2), pp. 239-250. 2013.

[5] PETROBRAS DISTRIBUIDORA S.A. LUBRAX UTILE DM (220, 320 e 460), 2011. Ficha de Informação de Segurança de Produto Químico - FISPQ. Disponível em<http://www.br.com.br/wps/wcm/connect/42aeac004 65fb146a6a5b6227c5c0cfd/fispq-lub-ind-diversaslubrax-utile-dm.pdf?MOD=AJPERES>. Acesso em 26 fev. 2015. 